\title{
Teorías sistémicas de la comunicación
}

\author{
Systemic Theories of Communication
}

\section{Dirk Baecker}

Departamento de Administración y Teoría Cultural, Universidad Witten/Herdecke, Alemania

\section{RESUMEN}

Las teorías sistémicas de la comunicación se derivan de la deconstrucción de la teoría matemática de la comunicación y del modelo de transmisión de Claude Shannon y Warren Weaver. Refiriendo a la pregunta irresuelta sobre la identidad de un mensaje para distintos observadores, estas teorías desarrollaron un modelo selectivo de comunicación. En función de superar el modelo ingenieril de la señalización (en vez de comunicación) propuesto por Shannon y Weaver, las teorías sistémicas no asumen que un mensaje es seleccionado de un set de mensajes posibles, sino que el set de posibles mensajes, así como el mensaje que será seleccionado de ese set (contexto), debe ser construido por los participantes de la comunicación.

PAlabRas Clave: Autología; Comunicación; Forma; Mensaje; Red; Observador; Selección; Sociedad; Teoría sistémica

\section{ABSTRACT}

Systemic theories of communication proceed from a deconstruction of Shannon's and Weaver's mathematical theory and transmission model of communication. Referring to the unresolved question of the identity of a message for different observers they instead develop a selection model of communication. In order to overcome the engineering model of signaling (rather than communication) offered by Shannon and Weaver systemic theories drop the assumption of a given set of possible messages any one message is considered to be selected from, and instead assume that the set of possible messages is to be constructed by the participants in communication as much as the single message then to be selected from that constructed set, also known as the context.

KEYWORDS: Autology; Communication; Form; Message; Network; Observer; Selection; Society; Systemic Theory

\section{TRANSMISIÓN}

Las teorías sistémicas de la comunicación se originan en la deconstrucción del modelo de trasmisión de Claude E. Shannon y Warren Weaver (1948): 
Fig. 1. Diagrama DE Un SISTEMA GENERAL DE COMUNICACIÓN

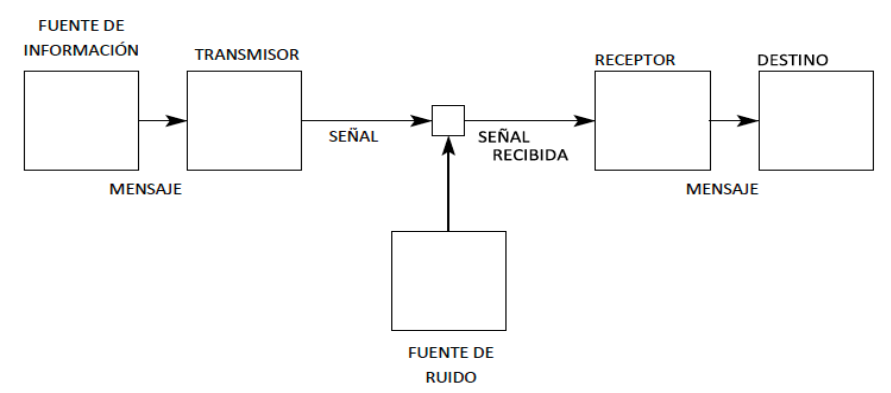

FUENTE: Shannon \& Weaver (1948: 7, 34)

Existen pocos modelos científicos que hayan causado más confusión que este. Aparece en la introducción de Weaver y en las páginas iniciales del primer capítulo de Shannon y su propósito parece ser el de permitirle al lector enfocarse en la pregunta ingenieril de cómo asegurar que una señal transmitida pueda ser recibida como la misma señal por un receptor, a pesar de la existencia de ruido proveniente de una fuente. La idea clave de Shannon fue usar conceptos de la mecánica estadística para asegurar que las posibles distorsiones por interferencia de una fuente de ruido pudieran ser corregidas por el receptor. El mensaje que llega al receptor se entendió como si este fuese: "seleccionado de un set de posibles mensajes" (Shannon \& Weaver 1948: 31), de modo de que el receptor solo tuviese que calcular la probabilidad de los mensajes posibles para así sustituir uno probablemente distorsionado por otro probablemente correcto.

La genialidad de Shannon reside en que descartó cualquier forma de conocimiento sustancial del 'significado' del mensaje por parte de los receptores, permitiendo que, por el contrario, estos se enfocaran en la información entregada por el mensaje frente a otros mensajes ya recibidos o aún esperados. Por ello, no hay nunca un solo mensaje, sino que siempre son muchos, y todos ellos pertenecen a un "set de posibles mensaje" (Shannon \& Weaver 1948: 31), pues uno es determinado por la probabilidad de todos los demás. Norbert Wiener, cercano colaborador de Shannon en ese tiempo, insistió en la importancia de esta idea. Wiener (1948: 46) recalcó que la cibernética tiene su punto de partida en el mismo concepto de la mecánica estadística que consiste en "la resolución de una contingencia compleja en una secuencia infinita de más contingencias especiales -una primera, una segunda, una tercera, y así- cada una con probabilidad conocida". Existe una entretenida paradoja escondida en esta fórmula, a saber, que la 
suma de la probabilidad cero deriva en la probabilidad uno (Wiener 1948: 46s), pero no la revisaremos aquí.

Shannon enfatizó que esta "teoría matemática de comunicación" es relevante solo para comprender y resolver el problema ingenieril de la trasmisión de señales y rechazó las implicancias que pudiera tener la solución de este problema para la comprensión de los 'aspectos semánticos de la comunicación'. De hecho, originalmente no presentó 'la', sino 'una' teoría matemática de comunicación (Shannon 1948), dejando la selección del artículo definitivo y más osado a Weaver, quien armó el libro gracias a los artículos de Shannon. Sin embargo, la reticencia de Shannon de generalizar su teoría no evitó que sus ideas, y las de Weaver, respecto de la 'comunicación' fueran rápida y entusiastamente aprehendidas, como también efervescentemente rechazadas por científicos sociales (v. gr. en lo que respecta a recepción, Jakobson 1981; Bense 1969; y respecto al rechazo, Hayles 1999). Lo mismo le ocurrió a Wiener. Este rechazó la aplicación de la cibernética a las ciencias sociales por la insuficiente cantidad de proyecciones de datos estadísticos que describieran estos sistemas (Wiener 1948:24s), pero dicha advertencia no pudo evitar que estas ideas sobre 'comunicación y control', basadas en un cálculo de contingencias, se volvieran populares en varias áreas de las ciencias sociales, junto con el modelo de Shannon y Weaver.

El problema del diagrama de Shannon y Weaver es que 'transmisor' y 'receptor' son caracterizados como 'emisor' y 'receptor' de mensajes y representan así la comunicación como transmisión de mensajes de una persona a otra usando un canal, en el cual, a pesar de los problemas para la elección del código correcto para codificar y decodificar mensajes o que estos posiblemente estén distorsionados por ruidos desconocidos, de todos modos permite a los mensajes viajar de forma ordenada de un punto al otro. Los dos cuadros que representan la 'fuente de información' y el 'destino' fueron desechados, como referencias enigmáticas, posiblemente a la 'conciencia' de las personas involucradas en la comunicación.

Vale la pena señalar que Weaver hizo todo lo posible para fomentar el malentendido, sosteniendo, por ejemplo, que la "comunicación es uno de varios "procedimientos mediante los cuales una mente afecta a otra" (Shannon \& Weaver 1948: 3) y, a diferencia de Shannon, se refirió no solo a problemas 'técnicos' sino también 'semánticos' y de 'efectividad' (Shannon \& Weaver 1948: 4). Incluso consideró a los mensajes "deseados" como selecciones del set de mensajes posibles (Shannon \& Weaver 1948: 7). Weaver 
consideraba importante retomar este último punto, porque podía mostrar un camino trascendente a la selección y los cálculos estadísticos, un camino hacia los 'deseos', propósitos, metas y motivos del receptor. No por último, enfatizó en que la advertencia de Shannon sobre la irrelevancia del aspecto semántico para lo ingenieril no significaba necesariamente que los problemas ingenieriles fuesen irrelevantes para la semántica (Shannon \& Weaver 1948: 8).

Creo que fue correcto el énfasis de Weaver en este punto, sin embargo los problemas ingenieriles no son relevantes para la semántica, pues la ingeniera se ocupa solo de mensajes como señales que viajan por un canal. Estos se vuelven relevantes cuando consideramos la posibilidad de generalizar el modelo de comunicación inherente a la definición estadística de información de Shannon (Baecker 1997).

\section{EL OBSERVADOR}

En el libro escrito en conjunto, Shannon cita dos artículos en el primer párrafo de su capítulo, uno sobre la 'velocidad del telégrafo' (Harry Nyquist) y otro sobre la "transmisión de información" (Ralph Hartley), los cuales ya habían abordado la 'proporción-señal-ruido', por lo que solo creyó necesario agregar algunas ideas sobre el ruido y, nota bene, la estructura estadística del mensaje original. En lo que es posiblemente la más importante y engañosa afirmación del capítulo, escribe en el segundo párrafo: "El problema fundamental de la comunicación es reproducir en algún punto, ya sea exacta o aproximadamente, un mensaje seleccionado en otro punto" (Shannon \& Weaver 1948: 31). Se podrían escribir libros enteros sobre cualquiera de las palabras en esta cita, de hecho, así lo hizo Michel Serres (Serres 1968, 1980, 1982), pero solo nos enfocaremos en la 'reproducción'. Que el problema de la comunicación sea la reproducción puede ser el indicador más obvio de que la teoría lidia con el problema ingenieril de cómo asegurar que la señal enviada sea la misma señal recibida. De hecho, aun cuando no se cite literalmente, esta afirmación se volvió el mantra de los teóricos de comunicación que buscaban una manera de asegurar que la comunicación trasmitiese exitosamente el significado que el emisor tiene en mente al receptor que busca entender dicho significado. Mantenga el canal limpio, o mejor sin dominación (Habermas 1984), asegúrese que todos sean racionales y bien intencionados, y con certeza todo resultará bien. La comunicación será po- 
sible y podrá resolver el destino de la humanidad, o así continuaba el razonamiento.

El problema de esta lectura bien intencionada de la afirmación es que ignora algo que todo filósofo y científico cognitivo sabe: que las mentes y los corazones están clausurados entre sí (y si no también a ellos mismos) (solo véase Locke 1959, vol. 11: 8-14), que la comunicación viene a reemplazar al entendimiento (esto es, a hacerlo posible, aunque el precio sea la mendacidad o la decepción) (Scheleiermacher 1995; Schlegel 1997), y que las preguntas fascinantes surgen cuando comenzamos a cuestionarnos sobre el modo en que las mentes (y los cuerpos) participan en la comunicación (luego de revisar las investigaciones sobre neurofisiología de Peter Müller o similares, véase Nietzche 2006). Peor aún, esta lectura ignora un problema conceptual más fundamental implicado en la afirmación. La 'reproducción' de un mensaje que ha sido seleccionado en un extremo en el otro significaría que alguien conoce el mensaje si verifica su idéntica reproducción. ¿Quién está en dicha posición? ¿Quién puede afirmar que el mensaje reproducido aquí es idéntico al seleccionado allá? Ciertamente no es ni el emisor ni el receptor, si por un momento defendemos esta confusa personalización, ya que ambos conocen solamente sus propias selecciones. Podría hacerlo un observador externo, pero ¿quién es este observador y cómo se involucra en la trasmisión de información? ¿Acaso necesitamos una autoridad divina o secular inventada precisamente con el propósito de decirnos que hemos reproducido de manera correcta nuestros mensajes y que nos entendimos bien los unos a los otros?

Nuevamente, la genialidad de Shannon consiste en anticipar el problema mismo de la imposibilidad de verificar la identidad de los dos mensajes -el primero seleccionado en un punto, el segundo en otro- transmitidos mediante un canal posiblemente distorsionado y asumiendo la posibilidad de que el receptor sea incapaz de enfrentar la decodificación. De hecho, Shannon manifiesta explícitamente el problema sin ofrecer una solución. En el segundo capítulo, donde introduce el concepto de canales con ruido, nos presenta un 'sistema de corrección' que introduce al observador como una innovación clave (Shannon \& Weaver 1948: 68): 
FIG. 2. ESQUEMA DE UN SISTEMA DE CORRECCIÓN

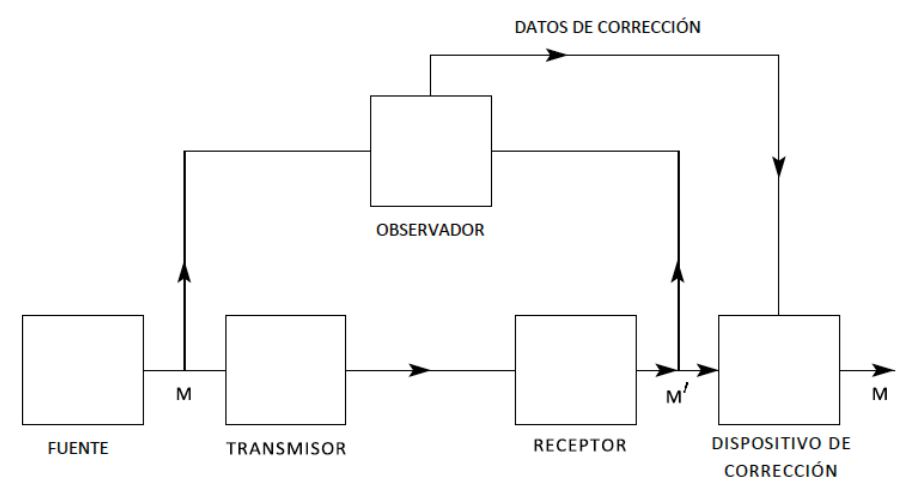

Este es un excelente ejemplo de auto-deconstrucción de un modelo, si es que puede hablarse de uno. El aspecto más interesante, aparte de la introducción del observador, es la corrección de M' a M mediante un 'aparato corrector' operado por el 'observador'. Sin embargo, la M corregida abandona luego el diagrama por la derecha y sale de la página sin la indicación de quién podría estar interesado en ella, ni quien es el receptor o si tiene un destino. Nótese que el enigma de este diagrama, al menos en parte, puede ser resuelto fácilmente cuando se observa el esquema de un 'sistema secreto general' que Shannon había generado en su artículo clasificado de 1946 'A Mathematical Theory of Cryptography' (desclasificado como Shannon 1949). Aquí (Shannon 1949: 661), el observador es el enemigo criptoanalista y la cuestión es cómo evitar se descifrado y lograr pasar el mensaje.

FIG. 3. ESQUEMA DE UN SISTEMA SECRETO GENERAL

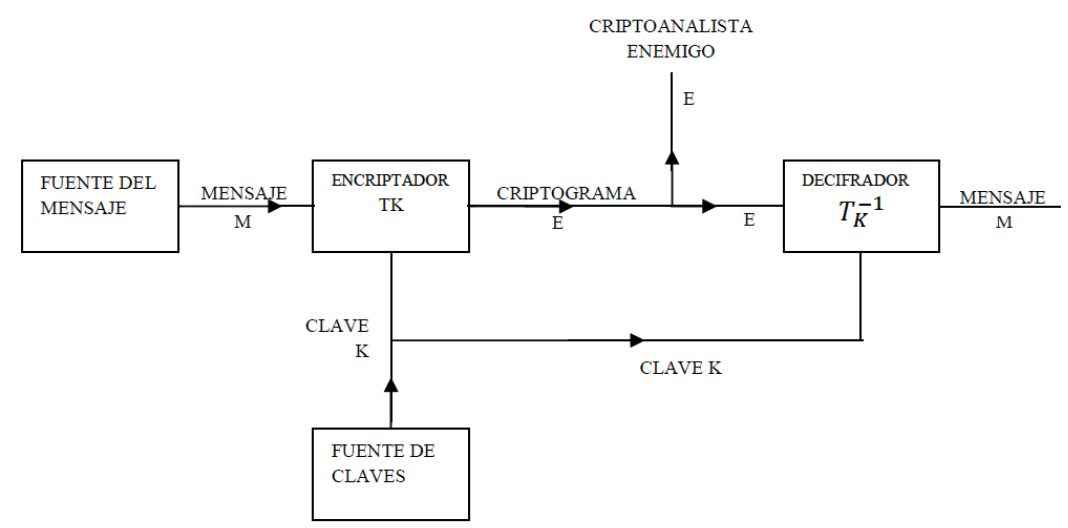

Debe notarse que la fuente de ruido ha desaparecido, dejando al observador en una posición en que puede determinar la diferencia entre M y M' sin tener que explicar necesariamente cómo ocurrió esta diferencia, ni tampoco la 
forma de detectarla. Es el 'observador' (ya sea él, ella o aquello) que determina y corrige la diferencia, y tiene los medios para hacerlo: un 'aparato de corrección' operando dentro del canal de transmisión para cualquiera que esté interesado en el resultado de sus operaciones.

La deconstrucción de la versión esquematizada del diagrama general del sistema de comunicación permite señalar que este presupone la identidad de dos mensajes que nadie en el sistema puede verificar, con la consecuencia de que se vuelve imposible conocer si ha ocurrido alguna transmisión y, menos aún, si ha acontecido la transmisión de un mensaje reproducido en un punto del modo en que este fue seleccionado en otro punto.

Se requiere un observador para conocer la diferencia entre el mensaje seleccionado y el mensaje reproducido. Se requiere un aparato corrector para corregir el mensaje distorsionado. Nadie sabe quién está involucrado en recibir el mensaje corregido o quién podría querer saber acerca del proceso completo de enviar, distorsionar y corregir el mensaje, excepto nuevamente el observador ominoso.

\section{SELECCIÓN}

Las teorías sistémicas de la comunicación reemplazan la trasmisión por la selección (MacKay 1969) y la identidad del mensaje por la recursividad (von Foerster 1980). Para hacer esto, introducen la noción de 'sistema' que es necesaria para mostrar cómo los mensajes se relacionan con la fuente de información, con el transmisor, con el receptor y con el destino a través de conexiones recursivas en lugar de conexiones causales (Pask 1970). El 'sistema' se distingue del 'entorno' que es un indicador de fuente de ruido. Por ello, la noción de canal involucra acoplamiento estructural, en vez de acoplamiento operacional, entre las distinciones que el transmisor y el receptor usan para codificar y decodificar mensajes. El acoplamiento estructural ocurre cuando unidades complejas se relacionan para lidiar con su propia complejidad; el acoplamiento operacional ocurre cuando las operaciones de un sistema reproducen recursivamente a ambos sistemas (Maturana 1975; Luhmann 1992a). El observador presentado en el sistema de corrección de Shannon se multiplica y distribuye a través del sistema. La noción técnica de set de mensajes posibles ('alfabetos') es considerada un caso particular de un caso más general de construcción, producción y reproducción sistémica de sets de posibles mensajes ('marcos') (Baecker 2005). 
Sin embargo, para sostener todo esto debe conservarse el concepto pionero de Shannon, a saber, la noción estadística de que la información es la relación de un mensaje con un set de otros posibles mensajes. La información contendida en cualquier mensaje está dada por el cambio en la probabilidad de la ocurrencia de todos los demás mensajes posibles en comparación con el primero. Este paso decisivo, desde una definición sustancial a una definición relacional de la información -en línea con la noción de Gottlieb Frege (1980) de que el significado de una palabra reside en la proposición que ocupa esa palabra, y con la noción de Ferdinand de Saussure (1986) de que los elementos semánticos del lenguaje son diferencias con otros elementos-, fue captado tempranamente por Gregory Bateson, quien realizó el primer intento de generalizar la teoría de Shannon, defendiendo que la comunicación no era transmisión de información sino "la creación de redundancia o de patrones" (Bateson 1972: 412; véase también Watzlawick et al. 1967).

El concepto relacional de información es de gran importancia porque refiere a la relación entre redundancia y variedad (o la proporción señalruido) producida por el sistema de comunicación y que, al mismo tiempo, lo guía. Un sistema de comunicación puede, por ello, ser definido en términos de los siguientes conceptos:

- Un mensaje es seleccionado de un set de posibles mensajes.

- Cualquier unidad que participa en el sistema de comunicación es un observador que ocupa sus propias distinciones para identificar mensajes y los sets de mensajes posibles de los cuales pueden ser seleccionados.

- El sistema de comunicación es producido por un mensaje identificado por una unidad participante en ese sistema y, selectiva y recursivamente, atribuido a otra unidad, a un set de mensajes y fuentes de ruido posibles, estos tres componentes (unidad, set de mensajes y fuentes de ruido) constituyen una red que posee límites.

- La reproducción del sistema consiste en lidiar con la variedad para que se mantenga la redundancia. Esta redundancia distingue al sistema de todo lo demás, esto es, del entorno. La variedad y la redundancia describen la relación de cualquier mensaje con el set de mensajes posibles distribuidos en la red de mensajes, unidades, sets de posibles mensajes y fuentes de ruido.

Esta definición no estipula qué clases de mensajes son producidos por el sistema, si acaso son gestos en expresiones faciales o corporales, imágenes dentro de otras imágenes, palabras dentro de oraciones, números dentro de 
cálculos, o cualquier mezcla de todo esto en una combinación multimedial. Nótese que una definición de "cosas" como acoplamientos estrictos entre elementos en un "medio" constituido de acoplamientos débiles de los mismos elementos (Heider 1926) encaja perfectamente con esta definición de comunicación sistémica, pero tiene la ventaja de no requerir la definición estadística de un set de mensajes posibles, o 'medio'.

Esta definición no indica qué clase de entidades o unidades participan mientras tengan la posibilidad de generar sus distinciones y seleccionar sus mensajes, sean estos espíritus, demonios, animales, plantas, humanos o máquinas. Se debe tomar una interesante decisión terminológica: hablar de 'entidades' significa asumir que con ellas ha ingresado algo sustancial a la comunicación, mientras que la noción de 'unidad' toma a estas mismas y las hace depender de otras unidades y de una clase común para que se conviertan en 'unidades'. Por una parte, estamos lidiando con una definición circular de comunicación y, por otra, con entidades que toman parte en la comunicación, pero que no pueden reducirse a dicha comunicación. Es por eso que Georg Simmel (1950), por ejemplo, propuso que los individuos tienen que ser en parte no-sociables para participar del proceso de socialización.

Adicionalmente, la definición expuesta de un sistema de comunicación está abierta a la emergencia de un sistema de comunicación; cualquier mensaje puede comenzar mientras exista un set de posibles mensajes del cual se pueda considerar que el mensaje inicial fue seleccionado y así luego más mensajes relacionados. Niklas Luhmann enfatiza que cualquier selección de información, expresión o comprensión puede dar inicio a una comunicación en tanto encuentre los dos elementos con los cuales sintetizarse, aun si este comienzo refiere necesaria y paradójicamente a comunicaciones previas (resaltando de esta manera su vínculo, y diferencia, con la 'sociedad') (Luhmann 1995). Nótese que esta idea combina una alta arbitrariedad de iniciativa con una fuerte restricción para la constitución. El arranque de la comunicación se debe a que la comunicación es su propio cuello de botella.

Finalmente, nuestra definición no estipula un balance específico entre redundancia y variedad; repetir de manera estricta casi el mismo significado es tan probable como la dinámica más flexible de encontrar significados diferentes en casi todas las ocasiones. La pequeña palabra 'casi' es decisiva, 
porque sin esta el sistema sería o una máquina o un caos, los cuales se definen por su carecer de posibilidades de selección.

La definición expuesta de sistema de comunicación es, por ello, autológica, porque es tan relacional como lo defina el sistema. Cumple con la exigencia de Heinz von Foesrter (1974) de que cualquier formalidad en la comunicación no debe contener símbolos que representen communicablia: no puede probarse la comunicación mediante la existencia de algo que requiera comunicación. En vez de ello, puede comprobarse que hay comunicación gracias a la comprobación de que es posible la producción y reproducción de mensajes. Esta producción y reproducción, que depende de la relación entre redundancia y variedad, o de señal y ruido, se llama 'sistema'.

Si la definición de un sistema de comunicación no es sustancial sino relacional, otras dos características son esenciales. Las llamaremos 'recursividad' y 'socialidad'.

'Recursividad' significa que nunca habrá solo un mensaje a ser comunicado y nunca habrá solo una unidad de comunicación participando, sino que siempre será una cantidad indefinida de mensajes y un indefinido número de unidades, a pesar que lo más estructurante y que más cultiva la comunicación (vale decir, construir expectativas para producir una 'estructura', y valorar y revalorar expectativas para constituir una 'cultura') consiste en restringir el número de posibles mensajes y unidades. Por ello, es interesante ocupar las matemáticas de las funciones recursivas, incluyendo las dinámicas no lineales, para comprobar la posibilidad general de la comunicación en términos de valores-propios (eigen-values) o atractores que aparecen en dichas recursiones. Lo que originalmente se llamó 'objetos' y 'sujetos' de la comunicación, el propósito y los marcos, y definitivamente el set de posibles mensajes, son ejemplo de estos valores-propios (von Foerster 1974, 1980).

'Socialidad' significa que la interacción de las unidades participantes en la comunicación como observadores realizando sus distinciones y selecciones, se caracteriza tanto por independencia como por dependencia de y con estas unidades. Cualquier comunicación (producción de un mensaje en relación con otros posibles mensajes) necesariamente comienza con la aceptación de que otra unidad realiza selecciones respecto de sus propias distinciones y de que se tratarán de restringir dichas selecciones al ofrecer marcos que definan el set de posibles mensajes deseados por la primera unidad. Un sistema de comunicación, de la manera en que se definió aquí, 
presupone que las unidades participantes son libres de elegir si participan o no y sin embargo eligen participar. La comunicación presupone unidades que necesariamente deben comunicarse. Es por esto que la emergencia de estructuras y culturas debe hacer atractiva la participación, lo que significa que recluta unidades que participen en la comunicación y que hagan posible que en cualquier momento se pueda dejar la comunicación o darle un giro completamente inesperado que, sin embargo, debe estar conectado recursivamente.

Debido al principio de 'socialidad', Heinz von Foerster llama a que las teorías sistémicas de la comunicación acepten un principio hermenéutico básico: que el oyente, no el orador, determina la información del mensaje (von Foerster \& Pörksen 2002). Luhmann se refiere a la 'improbabilidad' de cualquier comunicación, cuya constitución social, vale decir, múltiple, la obliga a buscar, definir, negociar y mantener su atractivo para todas las unidades que participan por su cuenta, para lograr que una comunicación en general improbable se vuelva específica, situacional y temporalmente probable (Luhmann 1992b, 1995). La comunicación aparece si hay posibilidad de rechazarla o, demostrando indiferencia, evitarla, desatenderla e ignorarla.

\section{CORRECCIÓN DE ERROR}

Esta definición relacional, en vez de una sustancial, de la comunicación tiene la dificultad de determinar empíricamente si la comunicación ocurre o no. Ciertamente, ningún mensaje causa que el set de posibles mensajes del cual fue seleccionado sea producido, ni tampoco el siguiente mensaje que puede referir a este. Por ello, no hay posibilidad de comprobar la comunicación determinando empíricamente sus mecanismos causales, ya que no hay ninguno. Si es que hay algún mecanismo, es, nuevamente, uno relacional y de selección que implica el acoplamiento débil de organismos que siempre tendrán su rango de elección, y no un acoplamiento estricto que, como se ha visto, vincula causa y efecto.

Por ejemplo, Donald MacKay (1969) asume que hay mecanismos de comunicación que aseguran que cualquier comunicación refiera a los individuos que toman parte de esta, en tanto nunca existan cambios en el 'complejo-meta' (goal-complex) de una parte, provocado por la comunicación, que no estén vinculados con el cambio del 'complejo-meta' de la otra. Pero esto significa que la investigación empírica, tan importante para las ciencias ba- 
sadas en evidencias, se enfrenta a ciertas dificultades con la noción de comunicación. ¿Cómo puede uno determinar un cambio en el complejo-meta de un individuo? Ciertamente tampoco ayuda que las teorías de sistemas duden, desde las investigaciones epistemológicas de Gregory Bateson (1972), de la probabilidad empírica de causalidad, ya que cualquier causalidad depende de un observador que selecciona ciertas causas (pero ¿cuáles?) que causan (pero ¿cómo?) ciertos efectos seleccionados (nuevamente, ¿cuáles?). La preocupación provocada por esta objeción entre varias escuelas de investigaciones empíricas las lleva a insistir con más fuerza en evidencias que corroboren que las nociones teóricas refieren a alguna realidad.

Las teorías sistémicas de la comunicación no asumen que la comunicación sea algo que uno pueda indicar a observadores escépticos para que puedan 'verlo por sí mismos'. En vez de ello, el concepto de comunicación es un principio explicativo o "metadato" (Bagley 1968) que ayuda a ordenar la observación y generar marcos para realizar descripciones más ambiciosas y sofisticadas que indican la perspectiva de un observador respecto de una realidad compleja. Lo mismo ocurre con todos los demás conceptos en teoría de sistemas, empezando con 'sistema' y 'entorno', sin mencionar aún: 'función', 'límite', 'código', o 'forma'. Asumir que cualquier palabra, solo porque está ahí, debe referir a algo real sería caer preso de la "falacia de la reificación", como indicó Alfred North Whitehead (1967: 19s). De hecho, nuevamente, las palabras están determinadas relacionalmente, no sustancialmente.

Con todo, esto no significa que los teóricos de sistemas no estén interesados en la evidencia. La mera creencia es satisfactoria solo para resignarse. Las teorías de sistemas se preguntan por la evidencia, la que consiste en una descripción organizada sobre estos principios explicativos que evidencian más sobre el mundo y mejoran nuestra habilidad para lidiar con este. Pero ese criterio puede transformarse rápidamente en una ideología al servicio de sí misma, permitiéndole a una escuela celebrar sus procedimientos sin que nadie más comprenda cuáles podrían ser las ganancias. Por ello, incluso al trabajar en sus investigaciones, los teóricos de sistemas necesitan una pista más tangible para saber si van por el camino correcto o no. En su intento temprano de introducir el pensamiento de Shannon a las ciencias sociales, y de haberlo logrado con éxito en la psiquiatría, Jurgen Ruesch y Gregory Bateson fueron de los pocos en identificar esta pista. Es muy simple. Se preguntaron por la corrección del error. En cuanto un ob- 
servador puede observar dos unidades posiblemente involucradas en la comunicación, en que cada una refiere al comportamiento de la otra, dando cuenta primero de los errores y luego corrigiéndolos, se puede asumir correctamente que estas están involucradas en la comunicación. Por supuesto, este criterio supone que el observador propuesto por Shannon, que observa la 'trasmisión' de mensajes, se multiplique para que su posición describa la posición de cualquier unidad involucrada en la comunicación, incluyendo la de un observador externo involucrado en investigación.

\section{UNIDADES DE DIFERENCIA}

Concluiremos este trabajo con una mirada sobre algunos conceptos de comunicación que tienen la ventaja de estar en contacto con la definición relacional de información de Shannon y con la idea de Wiener de un sistema que refiere a muchas $-\mathrm{y}$ posiblemente vinculadas recursivamente- contingencias para enfrentar el mundo.

Los conceptos en cuestión son doble clausura, autopoiésis, sistema social, y forma. Cada uno de ellos enfrenta de manera diferente el desafío de cualquier teoría sistémica de la comunicación, a saber, combinar la operación de un mensaje que cambia el estado del mundo con la distribución de elementos que son al mismo tiempo producidos por dicha operación y necesarios para mantenerla.

Veamos primero 'doble clausura'. Este concepto fue introducido por von Foerster (1973) para describir la auto-organización de un sistema complejo que se reproduce operacionalmente (primera clausura) debido a que puede regular o programar cómo se lleva a cabo esta reproducción (segunda clausura). Mientras que la primera clausura elimina un margen de libertad en el sistema debido a que requiere que la finalización de una operación esté seguida por el inicio de otra, la segunda agrega un margen de libertad en la elección de estructuras y culturas para determinar la siguiente operación. Respecto de la comunicación, esto significa que los mensajes deben ser seleccionados efectivamente para reproducir el sistema, pero (en relación con la factibilidad de una selección) el sistema es relativamente libre de elegir el set de posibles mensajes desde el cual seleccionar. Los programas o -en términos más sociológicos- estructuras y las culturas que emergen describen qué ha sido probado como útil en la reproducción y qué es valorado y aun deseable por sobre otras posibles estructuras. Nótese que las posibles auto- 
descripciones del sistema están establecidas en el nivel de la segunda clausura, permitiendo el desarrollo de confusiones, ilusiones, ideologías, discursos y semánticas que toman una postura más bien simplista frente a la complejidad de la completa reproducción de operaciones en el nivel de la primera clausura. Debido a que la primera y la segunda están débilmente acopladas, es interesante para la investigación en comunicación inspeccionar la función de estructuras y culturas para compararlas, y, en su propia ilusoria y equivocada explicación, resaltar su contingencia.

El concepto de autopoiesis ideado por Humberto R. Maturana y Francisco J. Varela (1980) está íntimamente relacionado con esta idea de doble clausura. Distingue entre los elementos de un sistema que producen la red de elementos del sistema, por una parte, y la red de elementos del sistema, por otra. De esta manera, se puede marcar una distinción entre los elementos que reproducen operacionalmente al sistema, vía una 'clausura operacional', por una parte, y las 'estructuras' que contribuyen a esta reproducción mientras son materializadas de las más diversas formas empíricas, por otra. La reproducción operacional, esto es, la vinculación contingente de un elemento con otro para dar paso a un tercero, se mantiene inaccesible para el sistema como para el observador, porque involucra una auto-referencia que es todavía la suposición más axiomática de la teoría de sistemas. Aun así, la red que estructura la reproducción puede ser bien observada empíricamente. La red de elementos que juegan el mismo rol en la reproducción es accesible para un observador, quien puede incluso tratar de verse involucrado en esta reproducción.

En comunicación, la red consta de observadores y vínculos entre ellos que, debido a que pueden cambiar lo que el observador puede observar, pueden ser considerados observadores ellos mismos. Harrison C. White (1992) propuso un concepto de red correspondiente -aunque no haga referencia a las teorías sistémicas de la comunicación- que enfatiza que cualquier elemento de la red obtiene su identidad de los intentos exitosos y fallidos por controlar otros elementos. Aún más, las dinámicas generales de las redes son cambiar operaciones, ya que ningún elemento deja de obtener una perspectiva diferente sobre sí mismo y sobre los vínculos que lo conectan cuando se cambia a otro elemento o vínculo desde el cual observar la red.

El 'sistema social' es la versión de Niklas Luhmann de una teoría sistémica de la comunicación, muy en línea con las ideas de doble clausura y autopoiesis, pero mira más detenidamente las condiciones 'sociales' de un 
sistema de comunicación emergente. Se considera la 'comunicación' como el elemento básico del sistema, la cual emerge como una improbable y frágil síntesis de expresión, información, y comprensión, todas ellas atribuciones del sistema mismo, y que produce descripciones auto-simplificadas de 'acciones' (que incluyen 'individuos' y sus 'intenciones'), cuyas recurrencias son ocupadas para inferir 'estructuras' y 'culturas', 'tradiciones' y 'convenciones', cuando no el 'sistema' mismo. El mensaje, junto con el set de posibles mensajes y la selección de este set, es llamado "sentido", el cual sería la posibilidad de cambiar de un estado actual del mundo con sentido a otros estados del mundo posibles, también, con sentido (Luhmann 1990).

Al menos cuatro características aseguran la agitación interna del sistema social. Está, primero, la múltiple constitución de la comunicación que siempre refiere a varios participantes, quienes solo pueden ser tomados como tales artificialmente (esto es, una 'colectividad', por ejemplo, una 'clase' en una escuela) y todos quienes pueden retirarse en cualquier momento. Está, segundo, la auto-referencia de la comunicación que refiere siempre a un sí mismo elusivo. Tercero, está la naturaleza temporal de los elementos, los cuales aparecen y desaparecen como eventos, enfatizando la improbabilidad inherente de la comunicación y la necesidad de asegurar su continuación en cada momento.

La cuarta característica es la llamada 'sociedad'. "Sociedad" es el término de Luhmann (1997) que relata todo tipo de perturbaciones de y por la comunicación, las cuales son esperadas mientras la sociedad se organiza a sí misma en patrones y marcos recurrentes. Él escoge este término para dirigir la atención a la naturaleza reflexiva de una teoría de sistemas sociales, debido a que es una teoría elaborada dentro de la sociedad y que analiza la sociedad desde la distancia, a pesar de estar participando en ella. La teoría se desarrolla en la comunicación, a pesar que el autor y el lector puedan sentirse más bien solos en el mundo; por eso, debe exhibir algún entendimiento de la naturaleza de una sociedad que permita tal cosa, esto es, la escritura y la lectura de tal teoría. Citando a Warren McCulloch (1965; véase Luhmann 1997): ‘¿Qué cosa es una sociedad sino una teoría que la describe?'.

El término sociedad llama también la atención sobre el hecho que, contrariamente a una creencia bien extendida en las ciencias de la comunicación, no toda comunicación es cara-a-cara, masiva o estratégica, de manera que, aparte de la comunicación cara-a-cara, la mayor parte de la comu- 
nicación sería como "hablar al aire" y no solo sería transitoria sino también incorpórea y, por ello, de alguna manera de naturaleza inhumana (Peter 1999). Muy por el contrario, la mayor parte de la comunicación combina indexicalidad y contextualidad, referencias y participantes presentes y ausentes, cuerpos e ideas, historias y proyectos, dando un abundante espacio a distinciones para la investigación en comunicación entre interacción, organización, y sociedad. Si el cambio es ubicuo, debe aparecer primero el significado de las referencias actuales respecto de otras, ausentes o potenciales; por ello, se debe preguntar qué estructuras y culturas habilitan las agencias de comunicación, como familias, grupos de colegas, parejas, gente en una fila, oficinas, escuelas, ejércitos, partidos y otras para producir y manejar esta mixtura, entrecruzamiento y cambio.

Finalmente, el último concepto que captura la naturaleza, tanto operacional como distributiva de la comunicación, es la noción de forma de George Spencer-Brown (1969). La "forma" da respuesta a las preguntas sobre el modo en que una operación puede abarcar el foco en sí misma, cómo encuentra la siguiente operación, y cómo conoce el mundo donde tiene lugar. La respuesta a esta pregunta es que estamos lidiando con operaciones de distinción cuya forma podemos describir. Tienen un interior, un exterior, una línea que divide el exterior del interior y un espacio en donde ocurren. Por ello, cualquier operación de distinción con un-valor o, para citar a Gordon Pask (1981: 270, aunque refiere al 'entendimiento'), "sharp-valued", tiene, en su forma más simple, cuatro valores: lado indicado, exterior sin marcar, división y espacio. Hay otras formas posibles de forma que son más complicadas, ya que las distinciones pueden concatenarse de maneras distintas y también re-ingresar en ellas mismas, produciendo formas que producen y viven en funciones recursivas. El valor de la división, que no es parte de las cosas indicadas ni tampoco del exterior sin marcar, indica al observador que actualmente marca la distinción (Bateson 1972: 454-471) y, debido a ello, transforma todo el cálculo de forma en un cálculo de auto-referencia.

Luhmann (1997) introdujo esta noción de forma en la teoría de sistemas sociales y comenzó a reconstruir su teoría de la sociedad de acuerdo con este esquema. Con respecto a la comunicación, esto significa que cualquier comunicación puede ser considerada una operación de distinción (un mensaje selectivo) que indica algo como diferente de otra cosa, que puede o no ser especificada, de modo que operaciones posteriores de distinción 
puedan elegir afirmar o rechazar esa indicación, ya sea cruzando las distinciones para que observen su exterior o re-ingresando la distinción en sí misma para investigar al observador que marca dicha distinción, o acerca de la posibilidad de distinguir aquello que no ha sido previamente distinguido.

Usando la notación de Spencer-Brown, lo anterior se lee de la siguiente manera. Cualquier operación simple de comunicación (si es que es simple alguna vez) marca una distinción que indica en su lado interno un término distinguido, $m$ :

Comunicación (primer orden) $=\overline{\mathrm{m}}$

Esta comunicación atrae la atención de un observador de segundo orden (quien tal vez sea el primer observador observándose a sí mismo) ya sea en la indicación incluida, $m$, o en el exterior excluido de la distinción, el estado no marcado, $n$ :

Comunicación (segundo orden) $=\overline{\mathrm{m}} \mathrm{n}$

El observador de segundo orden que busca comunicación con el observador de primer orden, podría luego invitar a este último, o a algún tercero, a reflexionar sobre la posibilidad de distinguir $m$ de $n$ y así re-ingresar la distinción en la distinción.

Comunicación (re-ingreso) $=$

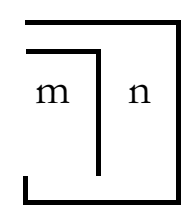

Nótese que el concepto de forma de Spencer Brown coincide perfectamente con el entendimiento relacional de la información propuesto por Shannon, ya que cualquier indicación (o mensaje) $m$ ha de ser considerado una distinción (o selección) de un set de otros posibles mensajes que pueden ser determinados, $n$, o dejados sin marcar. Con Spencer-Brown, sin embargo, la teoría matemática de la comunicación se vuelve auto-contenida. Cualquier distinción nos habla del observador marcándola, de un estado marcado indicado con esta y uno no marcado que necesariamente la acom- 
paña, de manera que el estado marcado puede en cualquier momento ser informado por la ignorancia que lo acompaña.

Las teorías sistémicas de la comunicación podrían considerar formas adicionales de comunicación, como las especificaciones operacionales, estructurales o culturales de la primera forma (Baecker 2005) y, así, aventurarse en un tipo de investigación empírica de comunicación que busca estar siempre en contacto con alguna complejidad ahí afuera y que busca reflexionar respecto a las propias distinciones que está ocupando.

Si las teorías sistémicas de la comunicación consisten en deconstruir el modelo de trasmisión de comunicación, enfatizando en el modelo de la selección en su lugar, y si la teoría de la mecánica estadística de la comunicación puede ser formalmente generalizada en favor de una teoría sistémica de la comunicación mediante la suposición de un set de posibles mensajes, entonces el concepto de forma de Spencer-Brown parece un candidato apropiado para indicar lo que son las teorías sistémicas de la comunicación y acoger a la siguiente generación de modelos comunicativos. Esta 'forma' parece adecuada para modelar la operación, el contexto y el cambio, sin nunca perder vista la esquiva identidad del observador que realiza la operación. $\mathrm{M}$

\section{REFERENCIAS}

Ashby, W. (1958). Requisite Variety and Its Implications for the Control of Complex Systems. Cybernetica, 1(2). 83-99.

Baecker, D. (1997). Bringing Communication Back into Cybernetics. Systemica: Journal of the Dutch Systems Group, 11, 11-28.

Baecker, D. (2005). Form und Formen der Kommunikation. Frankfurt aM: Suhrkamp.

Bagley, P. (1968). Extension of Programming Language Concepts. Philadelphia, Pa: University of City Science Center.

Bateson, G. (1972). Steps to an Ecology of Mind. New York: Ballantine.

Bense, M. (1969). Einführung in die informationstheoretische Ästhetik: Grundlegung und Anwendung der Texttheorie. Reinbek bH: Rowohlt.

De Saussure, F. (1986). Course in General Linguistics. La Salle, Il: Open court.

Frege, G. (1980). Translations from the Philosophical Writings of Gottlob Frege. Oxford: Blackwell. Habermas, J. (1984). The Theory of Communicative Action. Boston, Mass: Beacon Press.

Hayles, K. (1999). How We Became Posthuman: Virtual Bodies in Cybernetics, Literature, and Informatics. Chicago, Il: Chicago UP.

Heider, F. (1926). Thing and Medium. In F. Heider, On Perception, Event Structure, and Psychological Environment: Selected Papers [Psychological Issues 1 (3)] (pp. 1-34). New York: International university Press, 1959.

Hirschman, A. (1970). Exit, Voice, and Loyalty: Responses to Decline in Firms, Organizations, and States. Cambridge, Mass: Harvard UP. 
Jakobson, R. (1981). Linguistics and Poetics. In R. Rudy (Ed.), Selected Writings, vol. III: Poetry of Grammar and Grammar of Poetry (pp. 18-51). The Hague: Mouton.

Krippendorff, K. (1994). A Recursive Theory of Communication. In D. Crowley \& D. Mitchell (Eds.), Communication Theory Today (pp. 78-104). Cambridge: Polity Press.

Locke, J. (1959). An Essay Concerning Human Understanding. New York: Dover.

Luhmann, N. (1990). Meaning as Sociology's Basic Concept. In, Essays on Self-Reference (21-79). New York: Columbia UP.

Luhmann, N. (1992a). Operational Closure and Structural Coupling: The Differentiation of the Legal System. Cardozo Law Review, 13, 1419-1441.

Luhmann, N. (1992b). What Is Communication? Communication Theory, 2, 251-259.

Luhmann, N. (1995). Social Systems. Stanford, Ca: Stanford UP.

Luhmann, N. (1997). Die Gesellschaft der Gesellschaft. Frankfurt aM: Suhrkamp.

MacKay, D. (1969). Information, Mechanism and Meaning. Cambridge, Mass: MIT Press.

Maturana, H. (1975). The Organization of the Living: A Theory of the Living Organization. International Journal of Man-Machine Studies, 7, 313-332.

Maturana, H. \& Varela, F. (1980). Autopoiesis and Cognition: The Realization of the Living. Dordrecht: Reidel.

McCulloch, W. (1965). Embodiments of Mind. Cambridge, Mass: MIT Press.

Nietzsche, F. (2006). On Truth and Lies in a Nonmoral Sense. In The Nietzsche Reader (pp. 114123). MaIden, Mass: Blackwell.

Pask, G. (1970). The Cybernetics of Behavior and Cognition Extending the Meaning of 'Goal'. Cybernetica 13, 139-159; 250.

Pask, G. (1981). Organizational Closure of Potentially Conscious Systems. In M. Zeleny (Ed.), Autopoiesis: A Theory of Living Organization (pp. 265-308). Amsterdam: North-Holland.

Peter, J. (1999). Speaking into the Air: A History of the Idea of Communication. Chicago, Il: Chicago UP.

Ruesch, J. \& Bateson, G. (1951). Communication: The Social Matrix of Psychiatry. New York: Norton.

Schlegel, F. (1997). On Incomprehensibility. In J. Schulte-Sasse (Ed.), Theory as Practice: A Critical Anthology of Early German Romantic Writings (pp. 118-127). Minneapolis: University of Minnesota Press.

ScheIeiermacher, F. (1995). Friedrich Schleiermacher's 'Toward a Theory of Sociable conduct', and Essays in its intellectual-Cultural Context. Lewiston, NY: Edwin MelIen Press.

Serres, M. (1968-1980). Hermès Vol. 1: La communication. Vol. 2: L'interférence. Vol. 3: La traduction. Vol. 4: Lo distribution. Vol. 5: Le passage du nord-ouest. Paris: Le Seuil.

Serres M. (1982). The Parasite. Baltimore. MD: Johns Hopkins UP.

Shannon, C. (1948). A Mathematical Theory at Communication. Bell System Technical journal, 27, 379-423; 623-656.

Shannon, C. (1949). Communication Theory of Secrecy Systems. Bell System Technical journal, 28: 656-715.

Shannon, C. \& Weaver, W. (1948). The Mathematical Theory of Communication. Urbana, Il: Illinois UP.

Simmel, G. (1950). The Sociology of Georg Simmel. Glencoe, Il: Free Press.

Spencer-Brown, G. (1969). Laws of Form. London: Allen \& Unwin.

von Foester, H. (1974). On Constructing a Reality. [Reprinted in: Heinz von Foerster, Understanding Understanding: Essays on Cybernetics and Cognition (pp. 211-227). New York: Springer, 2003] 
von Foerster, H. (1914). Notes on an Epistemology for Living Things. [Reprinted in: Heinz von Foerster, Understanding Understanding: Essays on Cybernetics and Cognition (pp. 247-259). New York: Springer, 2003]

von Foerster, H. (1980). Epistemology of Communication. In K. Woodward (ed.), The Myths of information, Technology and Post-industrial Culture (pp. 18-27). Madison, WI: Coda.

von Foerster, H. (1993). For Niklas Luhmann: 'How Recursive is Communication?' Reprinted in: Heinz von Foerster, Understanding Understanding: Essays on Cybernetics and Cognition (pp. 305-323). New York: Springer, 2003]

von Foerster, H. \& Pörksen, B. (2002). Understanding Systems: Conversations on Epistemology and Ethics. New York: Plenum Press.

Watzlawick, P. Beavin, J. \& Jackson, D. (1967). Pragmatics of Human Communication: A Study of interactional Patterns, Pathologies, and Paradoxes. New York: Norton.

White, H. (1992). Identity and Control: A Structural Theory of Action. Princeton, NJ: Princeton UP. Whitehead, A. (1967). Science and the Modern World. New York: Free Press.

Wiener, N. (1948). Cybernetics, or Control and Communication in the Animal and the Machine. Cambridge, Mass: MIT Press.

SOBRE EL AUTOR

Dirk Baecker. PhD en Sociología, Universidad de Bielefeld, Alemania. Profesor del Departamento de Administración y Teoría Cultural de la Universidad Witten/Herdecke.

\section{CONTACTO \\ Dirk.Baecker@uni-wh.de}

Recibido: abril 2017

Aceptado: agosto 2017

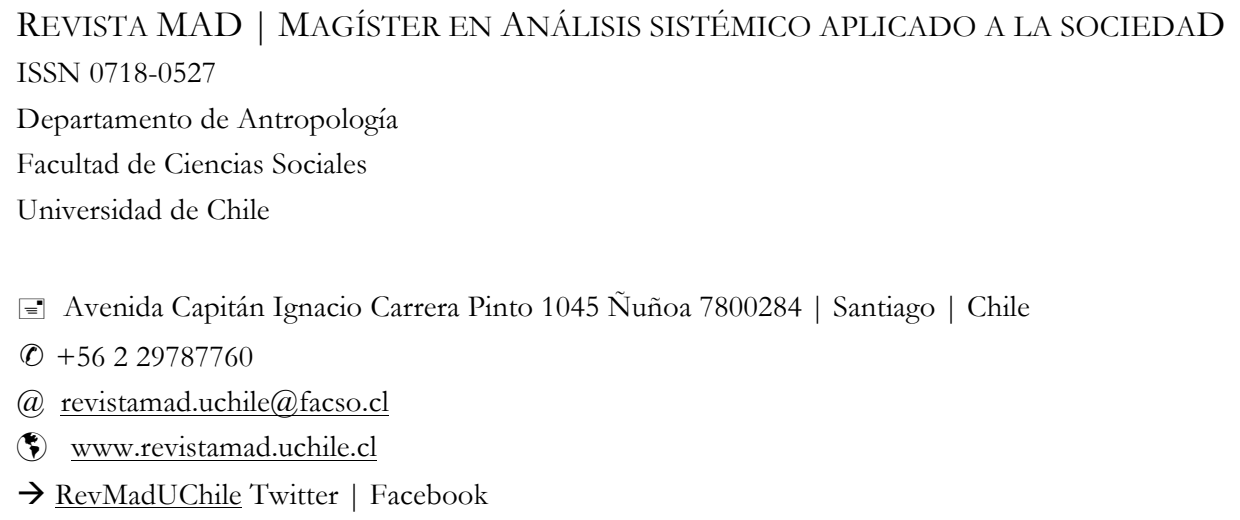

